\title{
Barium titanate/epoxy resin composite nanodielectrics as compact capacitive energy storing systems
}

\author{
G. C. Manika, G. C. Psarras* \\ Smart Materials \& Nanodielectrics Laboratory, Department of Materials Science, University of Patras, 26504 Patras, \\ Hellas (Greece)
}

Received 15 January 2019; accepted in revised form 2 April 2019

\begin{abstract}
Barium titanate/epoxy resin composite nanodielectrics were manufactured and their capability to store and harvest energy, upon request under DC conditions, was studied in this work. Morphological characterization in all nanocomposites was performed via scanning electron microscopy images and X-ray diffraction spectra, indicating the successful nanofiller's integration and dispersion within the polymer matrix. Applied DC voltage level varied from 10 to $240 \mathrm{~V}$ and the measurements were performed in the temperature range from 30 to $160^{\circ} \mathrm{C}$. Filler content enhances the energy efficiency of the manufactured systems, reaching the highest value of $58.2 \%$ for the $7 \mathrm{phr} \mathrm{BaTiO}_{3}$ nanocomposite. Increase of temperature results in an exponential decay of the coefficient of energy efficiency $\left(n_{\text {eff }}\right)$, indicating leakage currents' augment. DC and AC conductivity have been determined as a function of temperature for all nanodielectric systems. The temperature dependence of conductivity under DC and AC condition follows an Arrhenius form, which allowed the determination of activation energy in both cases.
\end{abstract}

Keywords: nanocomposites, $\mathrm{BaTiO}_{3}$, energy harvesting, coefficient of energy efficiency, charge transport

\section{Introduction}

There is an intense worldwide activity aiming to develop more sustainable energy conversion methods and technologies, in order to reduce the dependence on fossil fuels. The global air pollution, combined with the energy deficiency and the climate change leads to the replacement of fossil fuels energy resources with cleaner and renewable sources [1]. Considering the enormity of the environmental problem much effort should be carried out not only for the substitution of fossil fuel, but also for the improvement of the energy efficiency in current energy storing systems. Nowadays, a significant amount of the produced energy is lost in terms of heat, mainly in electric power generation and transmission. For instance, during the production of electric energy almost $60 \%$ of the energy extracted from power plants is lost as waste heat, whereas another $10 \%$ is lost at its transformation and transmission [2, 3]. Consequently, the improvement in the efficiency of energy usage is of crucial importance. The introduction of novel systems which can improve the efficiency of the energy storage, transmission and harvest upon request, aims to meet the challenges in the modern society. Energy harvesting refers to 'self-powered' devices that require no external power for operation, either continuous or punctuated. A wide range of electronic devices from portable electronics to hybrid electric vehicles demand high energy efficiency, long term autonomic operation, low cost, low weight and environmental friendly energy storage and harvest procedures. The priority in usage of electrical power is signified in many aspects by everyday life, as well as by the transmission and the effective commercialization of the renewable energy [4]. The amount of the needed electric power ranges to several orders of

${ }^{*}$ Corresponding author, e-mail: G.C.Psarras@upatras.gr

(C) BME-PT 
magnitudes, starting from a few $\mathrm{mW}$ in the case of smart-card devices and reaching several MW in the case of load-leveling stations [4]. Among energy technology systems, dielectric capacitors possess an intrinsic high power density due to their very fast energy uptake and delivery. Capacitors are important bulk or compact elements in electronic circuits and devices occupying more than $25 \%$ of the volume and weight of the devices. Polymer based capacitors exhibit a number of advantages including compact form, lightweight, flexibility, and high energy/power density [5]. Nanostructured materials have drawn the scientific research attention upon their appearance, due to their superior and unique performance and properties. The term nanodielectrics refers to dielectric materials which comprise entities with dimensions at the nanometric scale [6]. Polymer matrix nanocomposites belong to this category and attend enhanced technological and scientific interest, replacing conventional materials in a variety of applications. Polymers exhibit high dielectric breakdown strength along with low energy density, while ceramic fillers exhibit high values of dielectric permittivity and low breakdown strength. The combination of both materials results in a composite system with enhanced dielectric properties [7-9]. Polymer nanocomposites can be considered as a distributed network of nanocapacitors, which has the ability to store and harvest electrical energy. Moreover, the extended interface between the polymer matrix and the nanoinclusions plays a major role in the performance of the nanodielectric materials.

Epoxy resins are used as polymer matrices in many high-tech applications due to many factors such as their insulating nature, resistance to corrosive environments etc. In addition, ceramic nanoparticles such as the wide band gap barium titanate $\left(\mathrm{BaTiO}_{3}\right)$, are considered as very promising fillers being able to improve the dielectric properties and functionality of the nanocomposites, due to their high permittivity and temperature dependent polarization.

In this study nanocomposites constituted of an epoxy resin (ER) as a matrix and barium titanate $\left(\mathrm{BaTiO}_{3}\right)$ nanoparticles as the reinforcing phase, have been prepared and studied at various filler contents. Their morphological profile was examined via X-ray diffraction (XRD) and scanning electron microscopy (SEM). The produced nanocomposites were tested under AC and DC electric field and their ability to store and harvest energy was examined. The main goal of the present study is to determine the coefficient of energy efficiency of the $\mathrm{BaTiO}_{3}$ /epoxy resin nanocomposite system via the stored and harvested energy, with parameters the applied DC field, temperature and filler content. Furthermore, the DC and AC conductivity of the examined systems was studied as a function of filler content and temperature. The physical mechanisms, which are related to the conductance process were also studied and discussed. Finally, activation energies $\left(E_{\mathrm{A}}\right)$ were calculated by performing dynamic analysis in the $\sigma_{\mathrm{DC}}$ and $\sigma_{\mathrm{AC}}$ conductivity processes.

\section{Experimental section \\ 2.1. Fabrication of the nanocomposite materials}

Nanocomposite specimens were prepared with commercially available materials. A low viscosity epoxy resin (ER) (bisphenol-A), with the trade name Epoxol 2004A, and a slow reacting cycloaliphatic amine as curing agent, with the trade name Epoxol 2004B, were used for the preparation of the polymer matrix. Both reactants were provided by the Neotex S.A. Athens, Greece. Nanoparticles of barium titanate $\left(\mathrm{BaTiO}_{3}\right)$ were purchased by Sigma Aldrich (Burlington, Massachusetts, USA). According to the supplier's datasheet the diameter of the employed nanoparticles was less than $100 \mathrm{~nm}$. The preparation procedure was the following:

- Epoxy resin was mixed with the curing agent in a $2: 1(\mathrm{w} / \mathrm{w})$ ratio for the preparation of the matrix.

- With the matrix being in the liquid state, pre-calculated amounts of the ceramic filler were added.

- Stirring of the mixture at a slow rate under ultrasonication was performed to avoid the formation of clusters.

- The homogenized mixtures were poured in moulds and the curing process took place at ambient for a week, followed by post-curing at $100^{\circ} \mathrm{C}$ for 4 hours.

The $\mathrm{BaTiO}_{3}$ content of the prepared samples is expressed in parts per hundred resin $[\mathrm{phr}]$ per weight and the manufactured samples were: 0 (unreinforced epoxy), 1, 3, 7, 10, 12 and $15 \mathrm{phr}$.

\subsection{Methods}

\subsubsection{X-ray diffraction (XRD)}

For the XRD experiments a Bruker AXS D8 Advance (Coventry, UK) device with Bragg-Brentano geometry was employed. A LynxEye detector and 
$\mathrm{Cu} \mathrm{K} \alpha$ spectral line $(\lambda=1.54062 \AA)$ was used as the incident radiation. Scan mode was continuous, the step was $0.02^{\circ} 2 \theta$ and the scan speed was $0.5 \mathrm{~s} /$ step. Source slit was $0.6 \mathrm{~mm}$ while voltage and current were at $40 \mathrm{kV}$ and $40 \mathrm{~mA}$ respectively.

\subsubsection{Scanning electron microscopy (SEM)}

The morphology and the quality of the nanoparticle dispersion, as well as the existence of agglomerates and voids inside the polymer matrix, were investigated by SEM (Carl Zeiss EVO MA 10), (Jena, Germany).

\subsubsection{Broadband dielectric spectroscopy (BDS)}

The electrical characterization was conducted by means of broadband dielectric spectroscopy (BDS) in the frequency range from $0.1 \mathrm{~Hz}$ to $10 \mathrm{MHz}$, using an Alpha-N Frequency Response Analyzer supplied by Novocontrol Technologies (Hundsagen, Germany). The applied $V_{\text {rms }}$ was kept constant at $1 \mathrm{~V}$, while the temperature was controlled by Novotherm system with $\pm 0.1{ }^{\circ} \mathrm{C}$ accuracy. The dielectric cell was a two terminal BDS 1200 system, in which specimens were placed and isothermal frequency scans were performed from ambient to $160^{\circ} \mathrm{C}$ with a temperature step of $5^{\circ} \mathrm{C}$. AC dielectric measurements were conducted according to the ASTM D150 specifications.

\subsubsection{DC electrical measurements}

DC measurements were conducted by a High-Resistance Meter, DC (Agilent 4339B) (Hyogo, Japan). The experimental procedure includes an automatic process that allows a continuous control of measurements for the charging/discharging sequence. The applied voltage levels were: 10, 50, 100, 150, 200 and $240 \mathrm{~V}$. The applied temperatures were: 30,60 , 80,100 and $120^{\circ} \mathrm{C}$. Specimens were put between

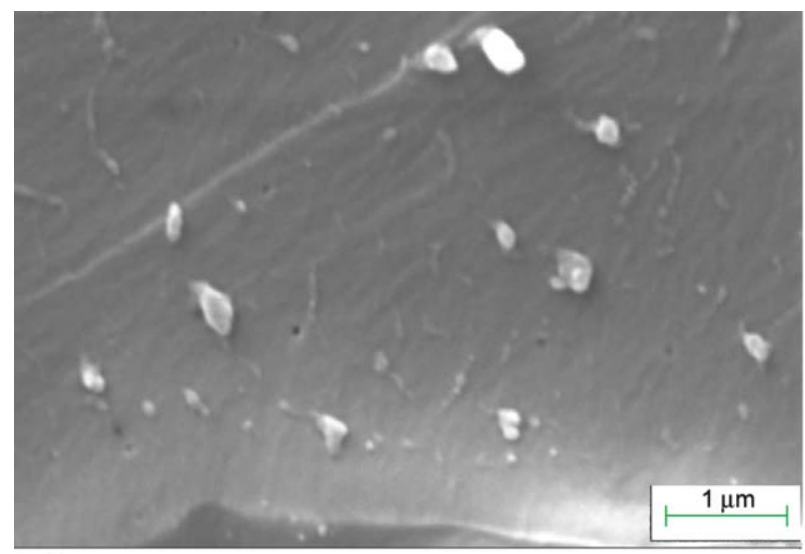

a)

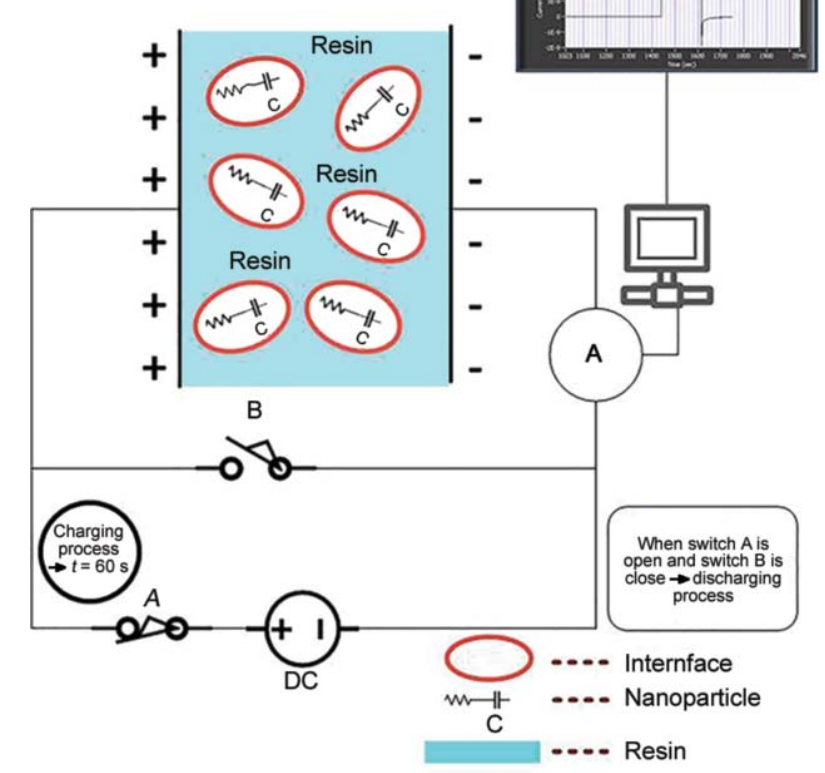

Figure 1. Schematic representation of the DC measurements.

two electrodes in a parallel-plate capacitor configuration. The charging time was $60 \mathrm{~s}$ for all the samples in every voltage level. Prior to every charging/ discharging sequence, it was ensured that no stored charges exist in the samples with the implementation of a discharging short-circuit procedure. Figure 1 shows a schematic representation of the experimental set up for the DC measurements. Measurements of the resistance in all samples were performed at 50,100 and $200 \mathrm{~V}$. DC measurements were conducted according to the ASTM D257 specifications.

\section{Results}

\subsection{Morphological characterization of $\mathrm{BaTiO}_{3}$ nanocomposites}

The morphology of the specimens was studied using XRD spectra and SEM images. Figure 2 presents

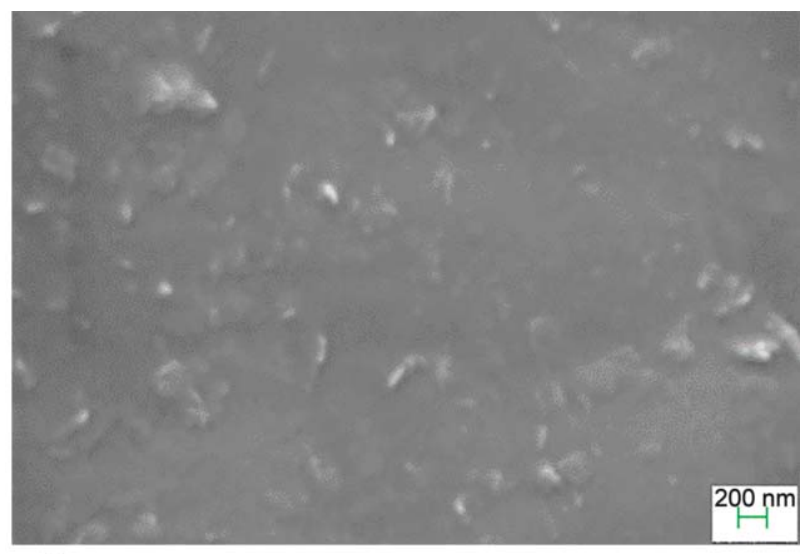

b)

Figure 2. SEM images for the nanocomposites with (a) 1 phr and (b) 15 phr $\mathrm{BaTiO}_{3}$ content. 


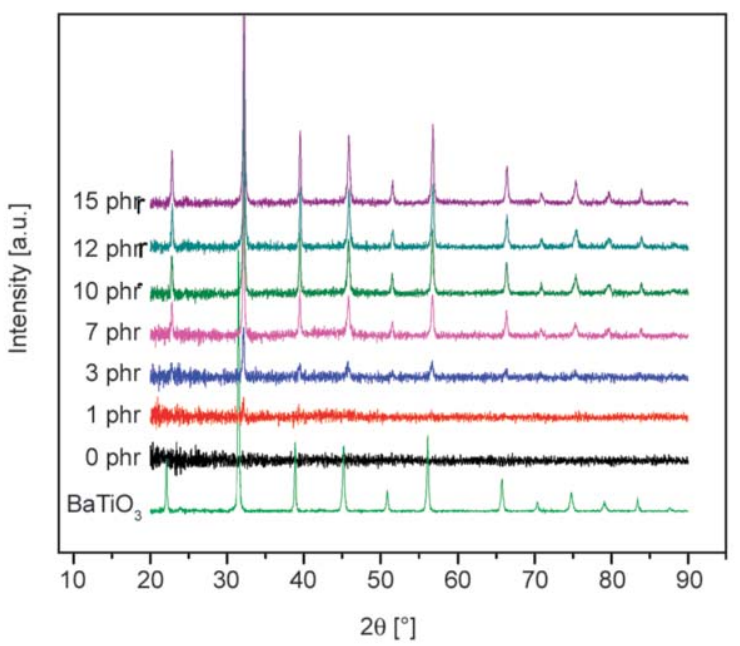

Figure 3. XRD patterns of all fabricated nanocomposites and $\mathrm{BaTiO}_{3}$ particles.

two representative SEM images of cryo-fractured

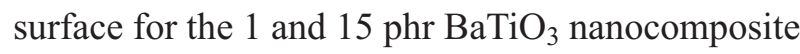
respectively. Obviously, the dispersion of the nanofiller in the polymer matrix can be considered as satisfactory, since fine nano dispersions can be observed. The respective XRD patterns are depicted in Figure 3. The amorphous state of the epoxy resin is evident because of the lack of sharp peaks. As the amount of the added nanoinclusions increases the peaks belonging to $\mathrm{BaTiO}_{3}$ become more intense, indicating a successful nanocomposite fabrication.

\subsection{Energy storage and harvesting/coefficient of energy efficiency}

The storing and harvesting procedures were operated by measuring the charging/discharging currents at various voltage levels and at different temperatures. As the applied DC field increases the current in both charging and discharging processes is larger. The discharging process takes place under no field application. All the specimens revealed similar behaviour. At the lowest applied DC voltage level of $10 \mathrm{~V}$ experimental noise was observed. The stored energy is a function of capacitance, which is determined by the geometry of the capacitor and the permittivity of the dielectric material. Equation (1) indicates that the only material property affecting capacitance is the dielectric permittivity [10-12]. The stored energy in the material-device is calculated by Equation (2):

$C=\varepsilon \cdot \varepsilon_{0} \cdot \frac{A}{d}$
$E=\int_{0}^{Q} V \mathrm{~d} q=\frac{1}{2} \cdot \frac{Q^{2}}{C}$

where $E$ is the capacitor energy, $Q$ is the accumulated charge, $C$ is the capacitance, $\varepsilon$ is the dielectric permittivity or dielectric constant, $A$ is the area of the capacitor's plate, $d$ is the distance of the capacitor parallel plates, and $\varepsilon_{0}$ is the dielectric constant of free space.

The stored and retrieved energy is evaluated via the charging and discharging currents for every temperature and every applied voltage. The energy can be calculated by integrating the $I=f(t)$ curves in Equation (3), and employing in each case the specimen's capacitance as determined by the dielectric measurements at the lowest measured frequency:

$E=\frac{1}{2} \cdot \frac{\left[\int I(t) \mathrm{d} t\right]^{2}}{C}$

Figures 4 and 5 depict the stored and harvested energy of the neat epoxy and the $10 \mathrm{phr} \mathrm{BaTiO}_{3}$ nanocomposite, at 30 and $80^{\circ} \mathrm{C}$ respectively.

The stored and harvested energy elevate with the increase of the applied field. Figures 4 and 5 show the efficiency of the storing/harvesting procedure at two temperatures and at all applied DC voltage levels. The introduction of a coefficient of energy efficiency considered to be necessary for the evaluation of the energy performance of the systems. The coefficient of energy efficiency ( $\left.n_{\text {eff }}\right)$ is defined as the ratio of the retrieved energy upon the stored one with parameters, the voltage, the temperature and the time [13] (Equation (4)):

$n_{\text {eff }}=\frac{E_{\text {discharged }}}{E_{\text {charged }}}$

Figure 4 and 5 indicate that the energy efficiency of the neat epoxy is very low compared with that of the $10 \mathrm{phr} \mathrm{BaTiO}_{3}$ specimen at ambient temperature. Moreover, with the elevation of the temperature $n_{\text {eff }}$ undergoes a large reduction for both epoxy and $10 \mathrm{phr} \mathrm{BaTiO}_{3}$ specimen. The same behaviour was observed in all studied nanocomposites. For comparison reasons, the stored and harvested energy values were calculated at the same time instant $(t=$ $10 \mathrm{~s})$. Table 1 lists the values of $n_{\text {eff }}$ for all studied specimens at $30^{\circ} \mathrm{C}$ and for three different applied voltage levels $(50,100,200 \mathrm{~V})$. 

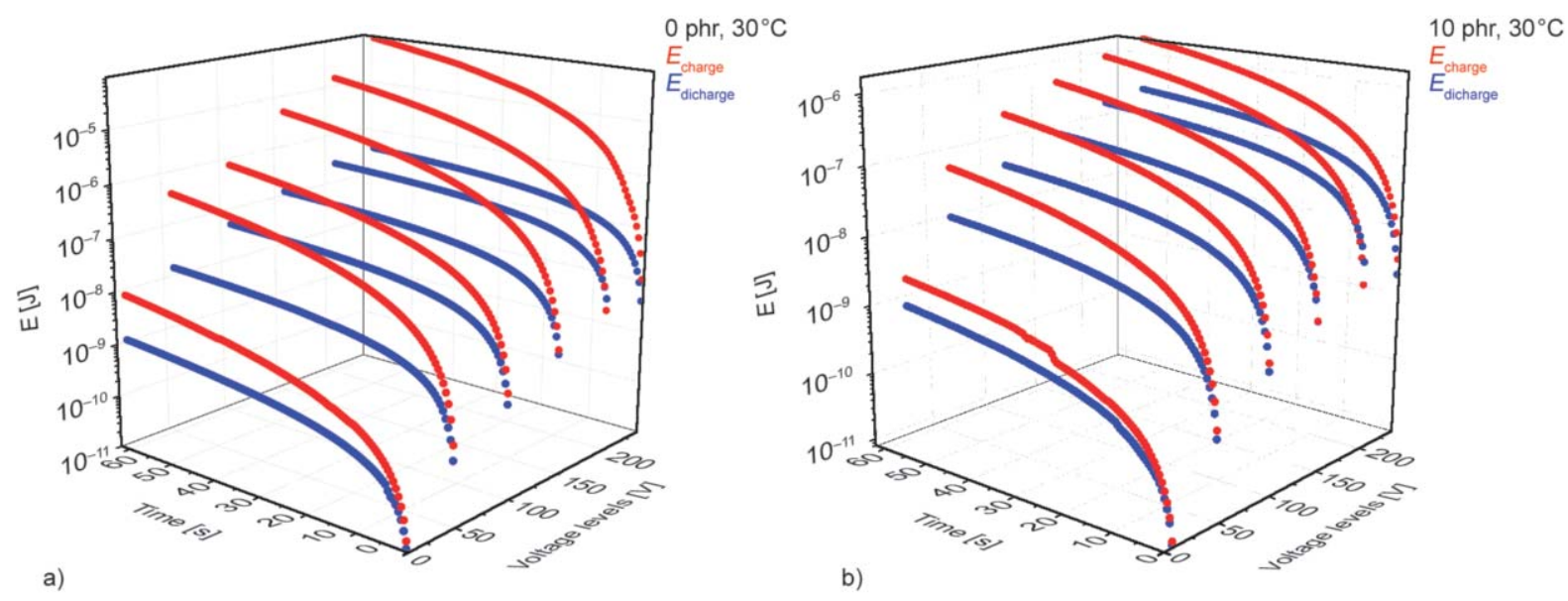

Figure 4. 3D spectra of stored and harvested energy as a function of time and $\mathrm{DC}$ voltage level at $30^{\circ} \mathrm{C}$ for (a) the neat epoxy and (b) $10 \mathrm{phr} \mathrm{BaTiO}_{3}$ nanocomposite.

Table 1. Coefficient of energy efficiency $\left(n_{\text {eff }}[\%]\right)$ at 50,100 and $200 \mathrm{~V}$ for the time instant $t=10 \mathrm{~s}$ at $30^{\circ} \mathrm{C}$, for all specimens.

\begin{tabular}{|c|r|r|r|}
\hline \multirow{2}{*}{$\begin{array}{c}\text { BaTiO }_{3} \\
\text { content } \\
\text { [phr] }\end{array}$} & \multicolumn{3}{|c|}{$\begin{array}{c}\boldsymbol{n}_{\text {eff }} \\
{[\mathbf{\%}]}\end{array}$} \\
\cline { 2 - 4 } & $\mathbf{5 0 ~ V}$ & $\mathbf{1 0 0} \mathbf{~ V}$ & $\mathbf{2 0 0} \mathbf{~ V}$ \\
\hline 0 & 17.11 & 28.02 & 13.23 \\
\hline 1 & 12.31 & 43.80 & 7.06 \\
\hline 3 & 58.16 & 55.83 & 54.56 \\
\hline 7 & 49.33 & 55.72 & 58.20 \\
\hline 10 & 41.86 & 39.84 & 50.42 \\
\hline 12 & 41.85 & 43.58 & 48.62 \\
\hline 15 & 38.33 & 39.68 & 50.52 \\
\hline
\end{tabular}

The $7 \mathrm{phr} \mathrm{BaTiO}_{3}$ nanocomposite exhibits the optimum energy performance at $200 \mathrm{~V}$ and $30^{\circ} \mathrm{C}$, with the value $n_{\text {eff }}=58.2 \%$. It is observed that at higher filler content $n_{\text {eff }}$ increases with the applied field. On the contrary, at lower filler concentrations, this tendency seems not to hold and the maximum $n_{\text {eff }}$ value is appeared at $50 \mathrm{~V}$ voltage level applied field. The coefficient of energy efficiency is a multi-parametric quantity affected by filler content, applied field, time and temperature. Moreover, the influence of a parameter cannot be described by a monotonous ascending or descending function, and thus optimum performance does not correspond, always, to the highest or the lowest value of the specific parameter. Field's intensity increases the number of charge carriers injected by the electrodes and also facilitates the transportation of space charges within the specimen by lowering the local potential barriers [14]. This is reflected in the increment of charging and discharging currents with the applied voltage, although a secondary influence of applied voltage is the strengthening of leakage currents. On the other hand, nanofiller content is responsible for the heterogeneity of the systems and the extended interface. Space charges migrate under the influence of the field and
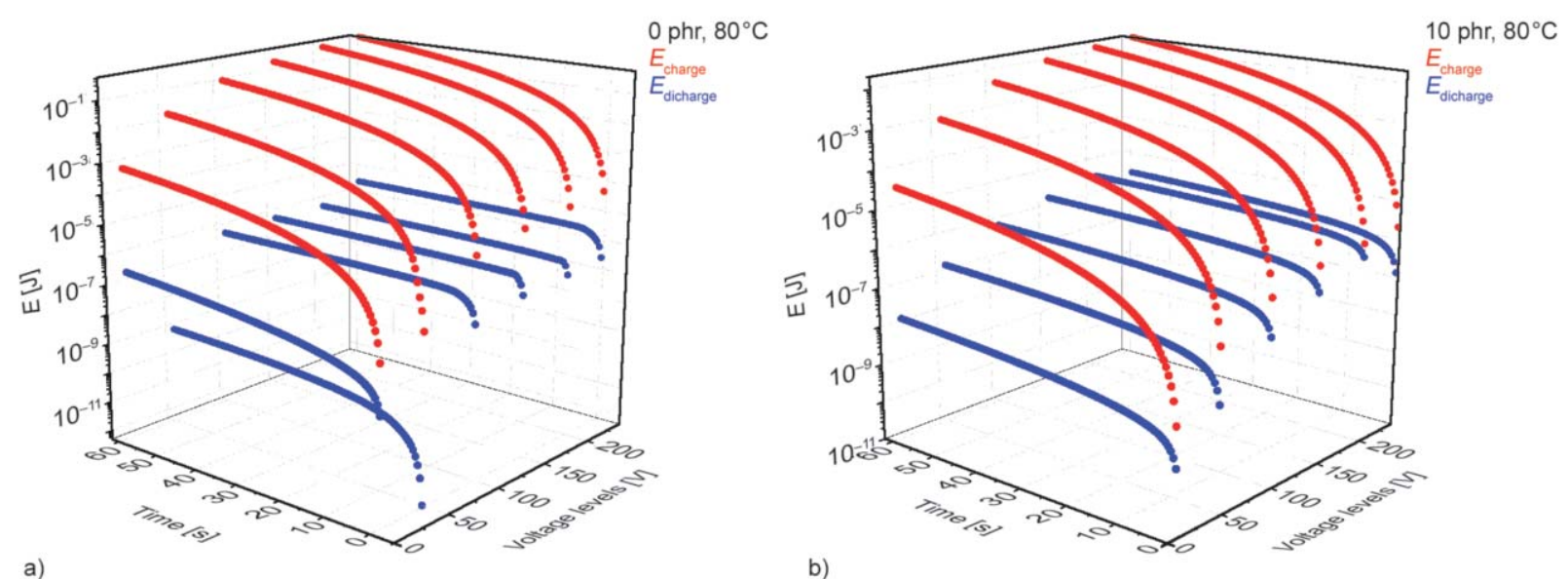

Figure 5. 3D spectra of $E_{\text {charged }}$ and $E_{\text {discharged }}$ as a function of time and DC voltage level at $80^{\circ} \mathrm{C}$ for (a) the neat epoxy and (b) the $10 \mathrm{phr} \mathrm{BaTiO}_{3}$ nanocomposite. 
accumulated at the interfaces, where they form dipoles with enhanced inertia [8]. This charge 'trapping' procedure at the interface contributes to the dielectric response and permittivity, especially in the low frequency and high temperature region. Charge carriers at the interface require receiving an amount of energy in order to overcome the potential barrier of their well and to participate in the conduction process. This activation energy can be acquired even by applying high strength fields or by elevating temperature. However, a minor number of space charges, even at low field and temperature, participates in the conduction process by migrating between adjacent conductive sites or by using interface as a conduction pathway [14]. The whole effect is known as interfacial polarization and its mathematical description includes two terms a dipolar and a conductive one [15]. Temperature provides thermal agitation to charge carriers influencing their mobility and resulting to higher values of conductivity, as shown in the next section.

\section{Discussion}

Figure 6 depicts the variation of coefficient of energy efficiency of the examined systems with temperature. It is apparent that the influence of temperature upon $n_{\text {eff }}$ in all nanocomposites is predominant. In particular, the increase of temperature leads to a dramatic decrease of $n_{\text {eff. Although, the absolute values }}$ of charged and discharged energy increase with temperature, the enhancement of conductivity is related to a significance augment of leakage currents. Leakage currents drain part of the stored charges from the nanocomposites, minimizing the beneficial effect of temperature on the harvested energy, numerator of Equation (4), while temperature maximizes the stored energy, denominator of Equation (4). The coefficient of energy efficiency as a function of temperature, $n_{\text {eff }}=f(T)$, appears to follow an exponential decay of the form expressed by Equation (5), where $A, B$ and $K_{0}$ are material's parameters.

$n_{\mathrm{eff}}=B+A \cdot \exp \left(-K_{0} T\right)$

Obtained values for the parameters of Equation (5) via fitting procedure are presented in Table 2, while Figure 6 depicts the variation of $n_{\text {eff }}$ with temperature, for all studied systems, at $200 \mathrm{~V}$ applied voltage. In the neat epoxy and the nanocomposite with $1 \mathrm{phr} \mathrm{BaTiO}_{3}$ the exponential fitting procedure with

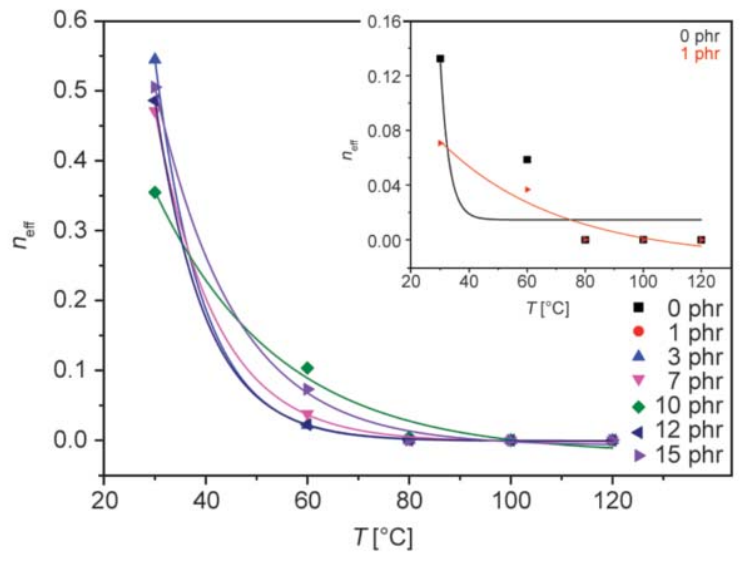

Figure 6. Coefficient of energy efficiency $\left(n_{\text {eff }}\right)$ at $200 \mathrm{~V}$ for time instant $t=10 \mathrm{~s}$, as a function of temperature for all specimens, inset shows data from the specimens with 0 and 1 phr $\mathrm{BaTiO}_{3}$ content.

Table 2. Obtained values of the parameters of Equation (5) as determined via the fitting procedure, for all specimens at $200 \mathrm{~V}$.

\begin{tabular}{|c|r|c|c|}
\hline \multirow{2}{*}{$\begin{array}{c}\text { BaTiO }_{3} \\
\text { content } \\
{[\mathbf{p h r}]}\end{array}$} & \multicolumn{3}{|c|}{$\boldsymbol{n}_{\text {eff }}=\boldsymbol{B}+\boldsymbol{A} \cdot \exp \left(-\boldsymbol{K}_{\mathbf{0}} \cdot \boldsymbol{T}\right)$} \\
\cline { 2 - 4 } & $\boldsymbol{A}$ & $\begin{array}{c}\boldsymbol{K}_{\mathbf{0}} \\
{\left[\mathbf{K}^{-1}\right]}\end{array}$ & $\boldsymbol{R}^{\mathbf{2}}$ \\
\hline 1 & 0.2 & 0.02 & 0.882 \\
\hline 3 & 13.2 & 0.10 & 0.999 \\
\hline 7 & 5.8 & 0.08 & 0.999 \\
\hline 10 & 1.3 & 0.04 & 0.981 \\
\hline 12 & 10.3 & 0.10 & 0.999 \\
\hline 15 & 3.5 & 0.06 & 0.998 \\
\hline
\end{tabular}

Equation (5) fails to describe satisfactorily the $n_{\text {eff }}$ values, deriving enhanced error. Both systems exhibit low conductivity and their electrical response is dominated by the insulating nature of the polymer matrix (Figure 6 inset).

\subsection{Conductivity/theoretical considerations and calculations}

Conductivity is one of the major electrical features of polymer nanocomposites exploited in a wide range of materials' applications. Moreover, conductivity values can vary over several orders of magnitude, extending from insulators up to conductors, depending upon the type and the amount of the nanocomposite constituents [16]. In our case, the semiconductive nanofillers have been used in combination with an insulating polymer matrix. Nanoparticles are able to form a network through the polymer matrix leading to higher conductivity values. These networks are usually formed during the manufacturing process. As the amount of reinforcing filler increases, the distance between the adjacent 
sites of the networks decreases, resulting in higher values of DC conductivity. In this study, DC and AC electrical conductivity was determined as a function of temperature for all the examined specimens. In the case of DC conductivity an additional parameter was the applied voltage level $(50,100$ and $200 \mathrm{~V})$. The DC electrical conductivity $\left(\sigma_{\mathrm{DC}}\right)$ was determined by Equation (6), in which $R$ is the volume resistance of the polymer systems, measured in the temperature range $\left(30-120^{\circ} \mathrm{C}\right)$, at $t=30 \mathrm{~s}$ instant of time:

$$
\sigma_{\mathrm{DC}}(T)=\frac{l}{A \cdot R(T)}
$$

where $l$ is the average sample thickness and $A$ the electrode surface. AC conductivity was determined according Equation (7) [8]:

$$
\sigma_{\mathrm{AC}}(T)=\varepsilon_{0} \cdot \omega \cdot \varepsilon^{\prime \prime}(T)
$$

where $\varepsilon_{0}$ is the permittivity of free space, $\omega$ the angular frequency of the applied field and $\varepsilon^{\prime \prime}$ the temperature dependent dielectric loss.

Figure $7 \mathrm{a}$ presents $\sigma_{\mathrm{DC}}$ as a function of temperature for the nanocomposites and the neat polymer matrix varying the $\mathrm{BaTiO}_{3}$ nanoparticles concentration at $50 \mathrm{~V}$ applied voltage. It is evident that all nanocomposites follow an exponential-like increase with a positive temperature coefficient, since $\mathrm{d} \sigma / \mathrm{d} T>0$. Inset in Figure 7a shows that the applied voltage does not affect the form of the $\sigma_{\mathrm{DC}}(T)$ function. The sign of the conductivity temperature coefficient is in accordance with the insulating or semiconductive nature of the examined materials and reflects the thermally stimulated random space charge migration within the nanocomposites [17].

In polymer matrix systems, potential barriers prohibit charges from migrating from site to site, thus it is necessary for the charges to acquire sufficient activation energy in order to overcome these barriers. The needed energy is exerted by the applied temperature. The temperature dependence of conductivity in dielectric materials follows the Arrhenius form expressed by Equation (8) [18]:

$\sigma_{\mathrm{DC}}(T)=\sigma^{0} \cdot \exp \left(-\frac{E_{\mathrm{A}}}{k_{\mathrm{B}} T}\right)$

where $\sigma^{0}$ is the pre-exponential factor, $E_{\mathrm{A}}$ is the activation energy, $T$ the absolute temperature and $k_{\mathrm{B}}$ is the Boltzmann constant. The DC conductivity values presented in Figure 7a lie within the range of semiconductive behaviour, approaching its lower edge [8]. Equation (8) is valid also for AC conductivity, at constant frequency of the applied field. Deviations from, Equation (8) in cases of AC conditions are related to dipolar effects occurring to polymer based systems, like glass to rubber transition [8].

$\mathrm{AC}$ conductivity is not only temperature but also frequency dependent as it is shown in Figure $7 \mathrm{~b} . \mathrm{BaTiO}_{3}$ as a wide band semiconductor exhibits low conductivity values, although higher than the epoxy matrix, and as a consequence the conductivity values of its nanocomposite systems persist low. It is apparent in Figure $7 \mathrm{~b}$ that at low frequencies, the temperature influence is more noticeable, revealing a thermally activated conduction mechanism. On the other hand, at high frequencies conductivity appears to be independent from temperature, varying exponentially

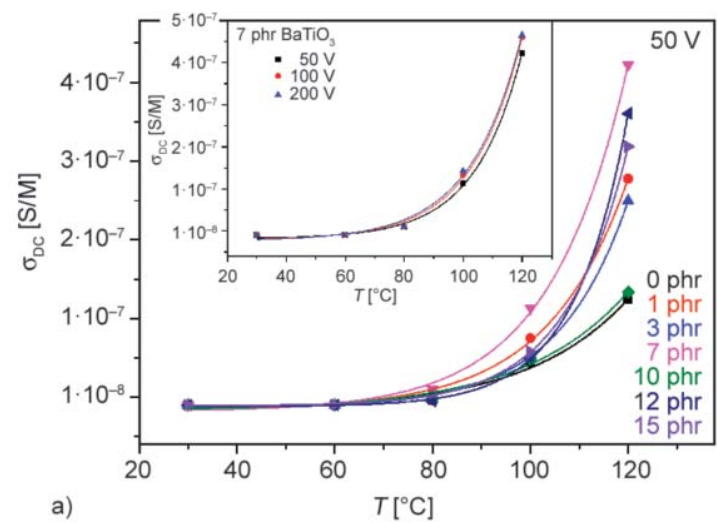

Figure 7. (a) $\sigma_{\mathrm{DC}}(T)$ for all specimens at $50 \mathrm{~V}$. Inset depicts the $\sigma_{\mathrm{DC}}(T)$ for the $7 \mathrm{phr} \mathrm{BaTiO}_{3}$ specimen for all applied DC fields $(50,100,200 \mathrm{~V}),(\mathrm{b}) \sigma_{\mathrm{AC}}(T)$ at $0.1 \mathrm{~Hz}$, inset shows the $\sigma_{\mathrm{AC}}(f)$ function for the $7 \mathrm{phr} \mathrm{BaTiO}_{3}$ specimen at several temperatures. 

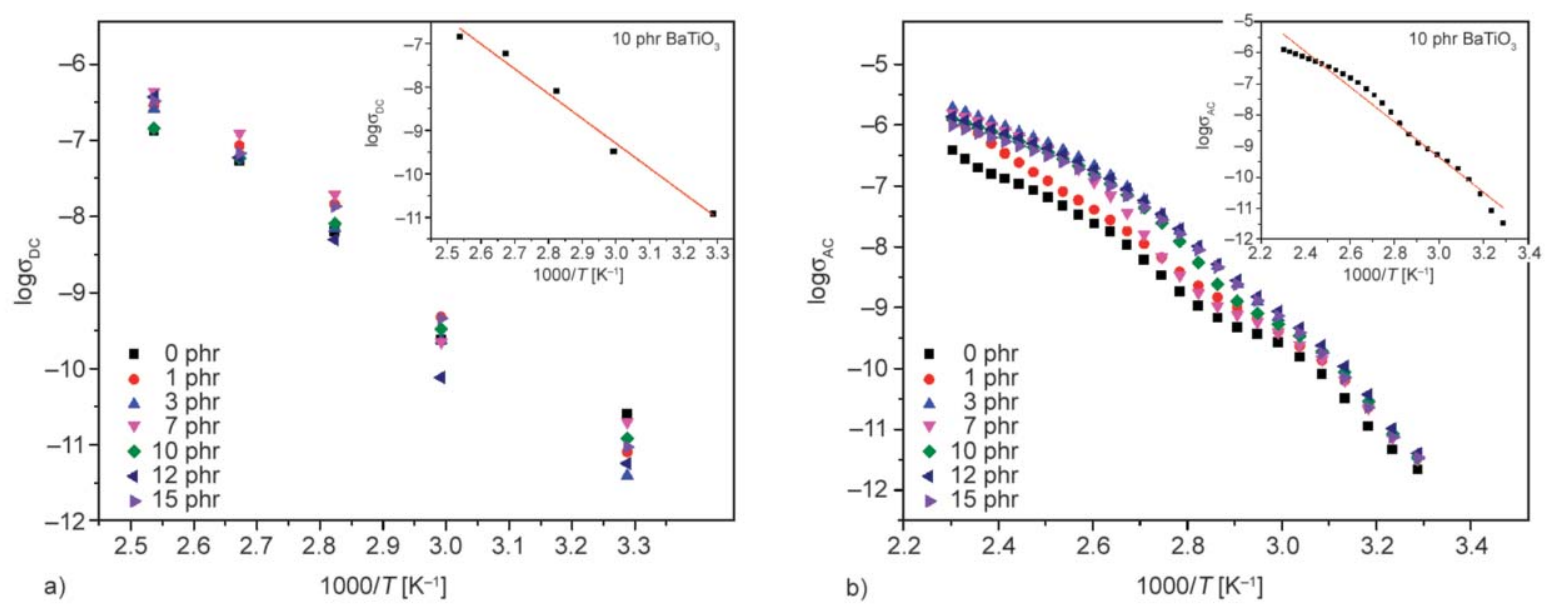

Figure 8. (a) DC conductivity at $50 \mathrm{~V}$ and (b) AC conductivity at $0.1 \mathrm{~Hz}$, versus reciprocal of temperature of all examined nanocomposite systems, inset shows the fitting processes for the $10 \mathrm{phr} \mathrm{BaTiO}_{3}$ nanocomposite in both cases.

with frequency, as shown in the inset of Figure $7 \mathrm{~b}$. In addition, in the low frequency limit, conductivity tends to attain constant values approaching its DC limit and the formed plateau increases with ascending temperature.

Figure 8 depicts the variation of conductivity with the reciprocal temperature in both DC and AC conditions, for all studied systems, respectively. Data are described via Arrhenius plots, since in their semilogarithmic representation, can be well fitted by linear regression, allowing the determination of the activation energy $\left(E_{\mathrm{A}}\right)$ for each nanocomposite. However, in the case of AC data, a small deviation from the completely Arrhenius behaviour is evident, probably because of the occurring dielectric relaxations.

Activation energy is a multi-parametric quantity, indicating the structure of the nanocomposites and the occurring interactions, being a function of the constituents' properties, the mean radius of particles or agglomerates, the mean interparticle separation and the extend of interface. Insets in Figure $8 \mathrm{a}$ and $8 \mathrm{~b}$, present representative examples of fitting data via Equation (8), for the nanocomposite with $10 \mathrm{phr}$ $\mathrm{BaTiO}_{3}$, in both $\mathrm{AC}$ and $\mathrm{DC}$ conditions. The determined values of activation energy, for the studied systems under DC and AC conditions, are shown in Table 3.

Obtained values of activation energy do not seem to follow a systematic pattern, although DC values are always higher than the $\mathrm{AC}$ ones. For the neat epoxy the determined activation energies in both $\mathrm{AC}$ and DC conditions do not seem to vary significantly. On the other hand, nanocomposites exhibit considerably higher values of activation energy determined under DC conditions comparing with the corresponding
Table 3. Activation energy of all $\mathrm{BaTiO}_{3} /$ epoxy specimens under both $\mathrm{AC}$ and $\mathrm{DC}$ conditions.

\begin{tabular}{|c|c|c|}
\hline $\begin{array}{c}\text { BaTiO }_{3} \text { content } \\
{[\mathbf{p h r}]}\end{array}$ & $\begin{array}{c}\text { AC conditions, } \boldsymbol{E}_{\mathbf{A}} \\
{[\mathbf{e V}]}\end{array}$ & $\begin{array}{c}\text { DC conditions, } \boldsymbol{E}_{\mathbf{A}} \\
{[\mathbf{e V}]}\end{array}$ \\
\hline 0 & 1.0267 & 1.0544 \\
\hline 1 & 1.0724 & 1.2476 \\
\hline 3 & 1.1521 & 1.3199 \\
\hline 7 & 1.1762 & 1.2301 \\
\hline 10 & 1.1223 & 1.1343 \\
\hline 12 & 1.0969 & 1.3381 \\
\hline 15 & 1.0932 & 1.2322 \\
\hline
\end{tabular}

ones in AC conditions, in general. AC conditions enhance charge carriers jumps between contiguous localized states leading consequently to lower values of activation energy compared to DC conditions. Under DC conditions charge carriers are imposed to overcome elevated potential barrier heights. Recalling that barium titanate is a wide band gap semiconductor, the influence of the $\mathrm{BaTiO}_{3}$ nanoparticles upon the overall conductivity of the nanocomposites and to the occurring charge transport mechanisms is not easy to be explained. Charge transport properties in polymer matrix nanocomposites are affected by several parameters, including the geometric characteristics of the nanoinclusions, the interaction between particles, the interactions between macromolecules and inclusions, the extent of aggregation of filler particles, the extend and properties of interface, the strength of disorder and the rheological behaviour of the matrix [8]. Particle-particle interactions and macromolecules-particles interactions constitute two competitive mechanisms influencing the mobility of polar parts of the polymer chains, interface response, and possibly trapping of charge carries at the systems' interface or isolated conductive sites $[19,20]$. 
Under this point of view, activation energy could vary with filler content reflecting the prevalent type of interactions, each time. Furthermore, DC fields force charges to migrate over long distances, resulting in higher $E_{\mathrm{A}}$ values in comparison with $\mathrm{AC}$ electric field. When the direction of the field remains constant, charge carriers' movement addresses difficulties raised by the insulating nature of the matrix. Thus, only a limited portion of charges is able to migrate, contributing to DC conductivity. The neat epoxy does not exhibit a notable difference in activation energy under AC and DC conditions, because of lacking the contribution of a more conductive phase. In the case of AC fields, charges move (hop) back and forth between adjacent sites of equilibrium, due to the alternation of the applied field. Possibly the number of charges participating in the process is higher than the corresponding under DC conditions, though the distances covered are significantly reduced.

\section{Conclusions}

In this work, nanocomposite systems consisting of an epoxy resin and barium titanate nanoparticles were fabricated and studied, varying the content of the reinforcing phase. Morphological characterization via XRD and SEM verified that nanocomposites were successfully fabricated. It was found that the prepared composite nanodielectrics can act as compact capacitive energy storing systems. The energy storage and harvest ability of the nanodielectrics were also investigated under DC conditions. Stored and harvested energy was determined at various temperatures by integrating the current-time function. It was found that the stored and harvested energy increases with applied DC voltage level. The coefficient of energy efficiency $\left(n_{\text {eff }}\right)$ increases with filler content at ambient conditions, reaching the highest value of $58.2 \%$ for the $7 \mathrm{phr} \mathrm{BaTiO}_{3}$ nanocomposite. Temperature imposes an exponential decay of $n_{\text {eff }}$ in all examined systems. AC and DC conductivity increases with temperature, being in accordance with the dielectric nature of the systems, implying at the same time thermally activated conduction mechanisms.

\section{References}

[1] Liu C., Li F., Ma L-P., Cheng H-M.: Advanced materials for energy storage. Advance Materials, 22, 28-62 (2010).

https://doi.org/10.1002/adma.200903328
[2] Martín-González M., Caballero-Calero O., Díaz-Chao P.: Nanoengineering thermoelectrics for $21^{\text {st }}$ century: Energy harvesting and other trends in the field. Renewable and Sustainable Energy Reviews, 24, 288-305 (2013). https://doi.org/10.1016/j.rser.2013.03.008

[3] Vouldis I., Millet P., Vallés J. L.: Novel materials for energy applications: A decade of EU-funded research. European Commission, Brussels (2008).

[4] Whittingham M. S.: Materials challenges facing electrical energy storage. MRS Bulletin, 33, 411-419 (2008). https://doi.org/10.1557/mrs2008.82

[5] Khanchaitit P., Han K., Gadinski M. R., Li Q., Wang Q.: Ferroelectric polymer networks with high energy density and improved discharged efficiency for dielectric energy storage. Nature Communications, 4, 2845/12845/7 (2013).

https://doi.org/10.1038/ncomms3845

[6] Psarras G. C.: Nanodielectrics: An emerging sector of polymer nanocomposites. Express Polymer Letters, 2, 460 (2008).

https://doi.org/10.3144/expresspolymlett.2008.55

[7] Pleşa I., Noţingher P. V., Schlögl S., Sumereder S. C., Muhr M.: Properties of polymer composites used in high-voltage applications. Polymers-Basel, 8, 173/1173/63 (2016).

https://doi.org/10.3390/polym8050173

[8] Psarras G. C.: Conductivity and dielectric characterization of polymer nanocomposites. in 'Physical properties and applications of polymer nanocomposites' (eds.: Tjong S. C., Mai Y-W.) Woodhead Publishing Limited, Cambridge, 31-69 (2010). https://doi.org/10.1533/9780857090249.1.31

[9] Barber P., Balasubramaanian S., Anguchamy Y., Gong S., Wibowo A., Gao H., Ploehn H. J., zur Loye H. C.: Polymer composite and nanocomposite dielectric materials for pulse power energy storage. Materials, 2, 16971733 (2009). https://doi.org/10.3390/ma2041697

[10] Imanaka Y., Amada H., Kumasaka F.: Microstructure and dielectric properties of composite films for embedded capacitor applications. International Journal of Applied Ceramic Technology, 8, 653-657 (2011). https://doi.org/10.1111/j.1744-7402.2010.02491.x

[11] Hao X.: A review on the dielectric materials for high energy-storage application. Journal of Advanced Dielectrics, 3, 1330001/1-1330001/14 (2013). https://doi.org/10.1142/S2010135X13300016

[12] Dang Z-M., Yuan J-K., Yao S-H., Liao R-J.: Flexible nanodielectric materials with high permittivity for power energy storage. Advanced Materials, 25, 6334-6365 (2013).

https://doi.org/10.1002/adma.201301752

[13] Manika G. C., Psarras G. C.: Energy storage and harvesting in $\mathrm{BaTiO}_{3}$ /epoxy nanodielectrics. High Voltage, 1, 151-157 (2016). https://doi.org/10.1049/hve.2016.0063 
[14] Dennison J. R., Brunson J.: Temperature and electric field dependence of conduction in low-density polyethylene. IEEE Transactions on Plasma Science, 36, 22462252 (2008).

https://doi.org/10.1109/TPS.2008.2003443

[15] Mathioudakis G. N., Patsidis A. C., Psarras G. C.: Dynamic electrical thermal analysis on zinc oxide/epoxy resin nanodielectrics. Journal of Thermal Analysis and Calorimetry, 116, 27-33 (2014).

https://doi.org/10.1007/s10973-013-3510-8

[16] Tsangaris G. M., Psarras G. C., Manolakaki E.: DC and $\mathrm{AC}$ conductivity in polymeric particulate composites of epoxy resin and metal particles. Advanced Composites Letters, 8, 25-29 (1999).

https://doi.org/10.1177/096369359900800104
[17] Wolpert D. Ampadu P.: Managing temperature effects in nanoscale adaptive systems. Springer, New York (2012).

[18] Psarras G. C.: Conduction processes in percolative epoxy resin/silver particles composites. Science of Advanced Materials, 1, 101-106 (2009). https://doi.org/10.1166/sam.2009.1015

[19] Vryonis O., Anastassopoulos D. L., Vradis A. A., Psarras G. C.: Dielectric response and molecular dynamics in epoxy-BaSrTiO 3 nanocomposites: Effect of nanofiller loading. Polymer, 95, 82-90 (2016). https://doi.org/10.1016/j.polymer.2016.04.050

[20] Sanida A., Stavropoulos S. G., Speliotis T., Psarras G. C.: Development, characterization, energy storage and interface dielectric properties in $\mathrm{SrFe}_{12} \mathrm{O}_{19}$ /epoxy nanocomposites. Polymer, 130, 73-81 (2017). https://doi.org/10.1016/j.polymer.2017.05.043 